\title{
Density of States in a Strongly Scattering 'Mesoglass'
}

\section{L.A. Cobus, W.K. Hildebrand, and J.H. Page}

University of Manitoba, Winnipeg, Manitoba Canada R3T 2N2 


\section{Density of States in a Strongly Scattering 'Mesoglass'}

ULTRASONICS

L.A. Cobus, W.K. Hildebrand, and J.H. Page

UNIVERSITY OF MANITOBA

University of Manitoba, Winnipeg, Manitoba Canada R3T 2N2

\section{Manitoba an}

\section{Introduction} media is important for obtaining a complete picture of wave transport. We present
such a robust procedure, which was used to detect the vibrational modes of elastic ultrasonic waves in a highly porous sintered glass bead network. Individual modes statistical approach was used to account for the possibility of missing or overlapping
modes enabling the density of states to be determined. In the intermediate frequenty modes, enabling the density of states to be determined. In the intermediate frequency
regime, the density of states was found to be independent of frequency. Level spacing regime, the density of states was found to be independent of frequency. Level spacing
statistics was also measured; level repulsion is seen, consistent with random matrix statistics was also
theory predictions.

\section{Mesoglass Samples
A 1:1 mixture of polydisperse glass and iron beads was sintered to create a
'mesoglass' material. The iron was then removed by etching, leaving a highly porous,
disordered structure.}

The sample material was cut into sufficiently
small pieces (typically $<1 \mathrm{~mm}^{3}$ ) so th small pieces (typically $<1 \mathrm{~mm}^{3}$ ) so that
individual vibrational modes may be resolve individual vib
and counted.

Three frequency
this material [1]:

1. Effective Homogenous Medium Regin

- Length scales larger than the disorder

the structure; the material behaves as
uniform medium with properties

2. Intermediate (Strong Scattering)
Regime - Length scales on the order of the
behaviour and very strong scattering occur

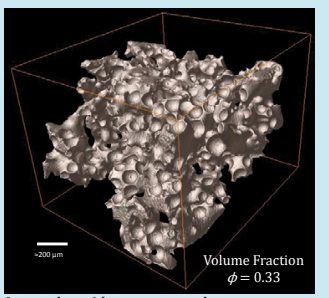

pore sizes of the material; fractal

Bulk Material Regime - Length scales smaller than the constituent parts of our

network; properties of the bulk medium (glass) dominate.

These length scales define the important frequency regimes in our medium.

Measuring Vibrational Modes

To measure the density of states, samples were placed between two transducers as

Meduce absorption.

-scattered

signals persist over
long times (right).

Modes are seen as peaks

in the Fourier transfort
of detected pulses.

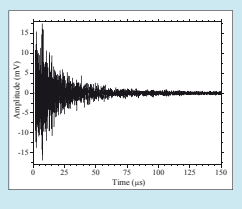

A reliable method of measuring the density of states in strongly scattering random

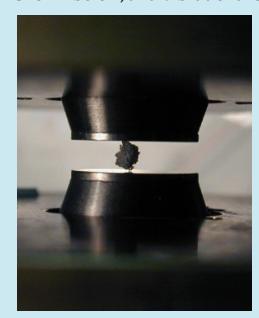

Detected pulses were averaged to improve resolution.

Mode Counting

The effect of varying contact points between

sample and transducers was studied (right).
Some of the same modes may be identified in

both spectra, but some are shifted or missing

The observed mode frequencies also vary
with the pressure between

transducers, as well as the vacuum pressure.

For a single sample orientation, ome can not
be confidident that all the modes are detected

within the frequency range of interest. Thi

statistical method.

Statistical Approach

By making many measurements, the density of states was determined using statistical

促

. Assume that for any one data set for a particular sample, there exists a probability $p$ of

random process, the Binomial distribution should be used as a parent distribution is a the number of modes observed.

The detection probability and total number of modes can then be written in terms of the

$$
p=\frac{\mu-\sigma^{2}}{\mu} \quad n=\frac{\mu^{2}}{\mu-\sigma^{2}}
$$

with corresponding wertainties.

\section{By making several measurements to find $\mu$ for each sample, and combining the results for}

Thresholding

When identifying peaks in the Fourier transform, a threshold was set to discern between

geneme resulting $\mu$ and $n$ plotted versus threshold. The correct
independent of $\mu$, enabling $n$ to be found unambiguously.

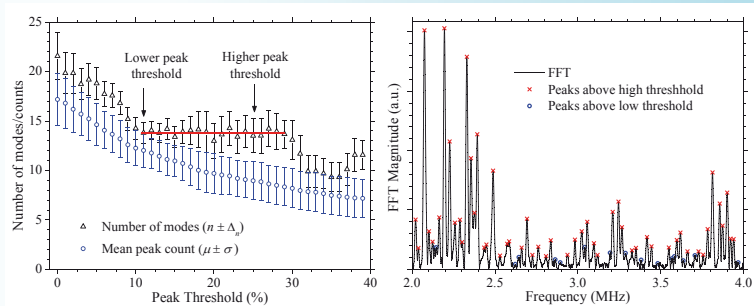

Left: The red line denontets she rerange where the modec counts ar
Right: Peaks found using the interactive thresholding method.

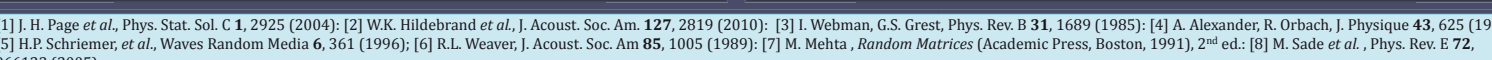

Density of States Results

The measured density of states is independent of freequency in the strong seattering
regime with this behaviour extending even into the effecective medium regime

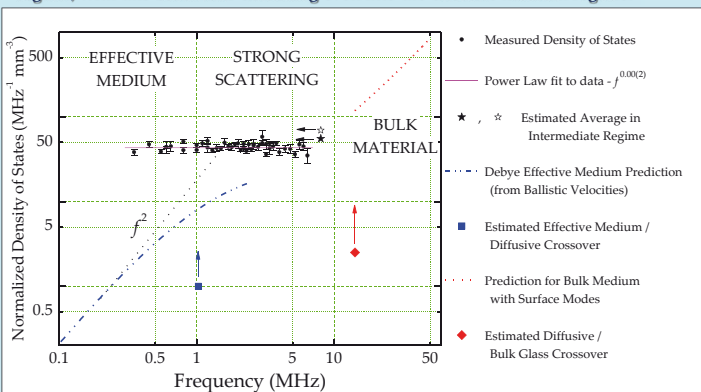

The power law fit can be interpreted using a fractal model $[3,4]$, which predicts a

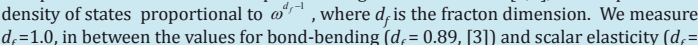

$4 / 3,[4])$ models. Our results are in good agreement with the value $d_{f}=1.05$ inferred
for sintered glass networks from low frequency velocity measurements $[5]$.

Level Spacing Statistics

S sured and compared with predictions from randon matrix theny. por a disordered system, we expect to see fluctuations consist showing level repulsion [6,7]. In a localized system, we expect a transition to a Poisso distribution near the mobility edge [8]. Our results are close to the predictions of
Gaussian statistics at low frequencies. At higher frequencies, the system behaviour appears to be between Gaussian and Poissonian statistics suggesting the approach to

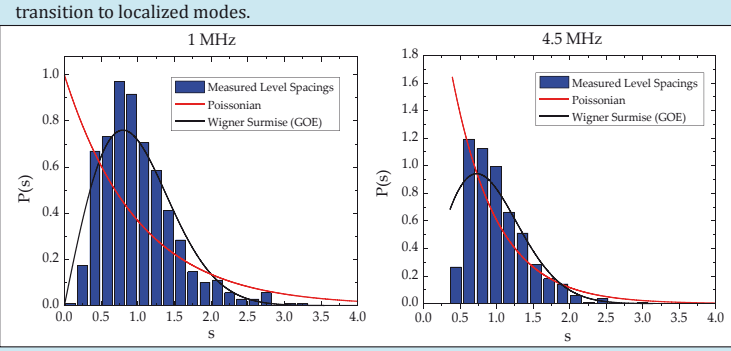

Conclusion

The density of states in a highly porous sintered glass bead network was measured. In The density of states in a highly porous sintered glass bead network was measured. In
the intermediate frequency regime, the density of states was found to be independent of frequency. Level spacing statistics show level repulsion, consistent with random matrix
theory predictions. These results suggest that Anderson localization may occur at theory predictions. These results suggest
higher frequencies near the upper crossover 